\title{
The aggregation of tuna around floating objects: What could be the underlying social mechanisms?
}

\author{
Robert Marianne ${ }^{a, b, c, *}$, Dagorn Laurent ${ }^{b}$, Deneubourg Jean Louis ${ }^{a}$
}

\begin{abstract}
a Unit of Social Ecology, Université libre de Bruxelles (ULB), Campus de la Plaine, Bd du Triomphe, Bat N0, N5, 1050, Brussels, Belgium

b UMR 212, Exploited Marine Ecosystems, Institut de Recherche pour le Développement (IRD), Avenue Jean Monnet, 34203 Sète Cedex, France

${ }^{\mathrm{C}}$ Institut français de recherche pour l'exploitation de la mer (Ifremer), Laboratoire de Technologie et Biologie Halieutiques, 8 rue Toullec, 56100, Lorient, France
\end{abstract}

\author{
*: Corresponding author: Marianne Robert, tel.: +33 (0)2 97873823 ; fax: +33 (0) 297873801 ; \\ email address : Marianne.robert@ifremer.fr
}

\begin{abstract}
:
Several empirical and theoretical studies have shown how the exploitation of food sources, the choice of resting sites or other types of collective decision-making in heterogeneous environments are facilitated and modulated by social interactions between conspecifics. It is well known that many pelagic fishes live in schools and that this form of gregarious behavior provides advantages in terms of food intake and predator avoidance efficiency. However, the influence of social behavior in the formation of aggregations by tuna under floating objects (FOBs) is poorly understood. In this work, we investigated the collective patterns generated by different theoretical models, which either include or exclude social interactions between conspecifics, in the presence of two aggregation sites. The resulting temporal dynamics and distributions of populations were compared to in situ observations of tuna behavior. Our work suggests that social interactions should be incorporated in aggregative behavior to reproduce the temporal patterns observed in the field at both the individual and the group level, challenging the common vision of tuna aggregations around FOBs. Our study argues for additional data to further demonstrate the role of social behavior in the dynamics of these fish aggregations. Understanding the interplay between environmental and social factors in the associative behavior of fish with FOBs is necessary to assess the consequences of the widespread deployment of artificial FOBs by fishermen.
\end{abstract}

\section{Highlights}

Identify which mechanisms are most likely to reproduce fish aggregations dynamics under FOBs. Dynamics and distributions of populations of behavioral models were compared to observations. Results challenge the common vision theses aggregations. Social interactions should be incorporated to reproduce the temporal patterns observed. - The characterization of these mechanisms is a priority for science-based fishery management. 
Keywords : Models Of Aggregation ; Monte Carlo multi-agents simulations ; Fish Aggregating Devices ; Social Behavior

\section{Introduction}

Understanding the spatiotemporal distribution and dynamics of wild animals remains a challenge for biologists. A particular question concerns the high densities of animals observed at certain specific points in space, e.g., aggregations (Camazine et al., 2001). A substantial literature is dedicated to the study of these patterns that appear in the wild for a wide range of taxa from bacteria to humans (Sumpter 2010). The ultimate behavioral causes of an aggregation can be very diverse and include feeding strategies, reproduction and resting (Krause and Ruxton, 2002 and Parrish and EdelsteinKeshet, 1999). Several lines of evidence indicate that the underlying mechanisms often result from the interplay between environmental and social factors (Canonge et al., 2011, Capello et al., 2011 and Camazine et al., 2001). To disentangle these driving factors (social and environmental), both experimental and modelling approaches are usually carried out. Through multi choice experiments (Canonge et al., 2011, Dussutour et al., 2004 and Jeanson et al., 2004a), the role of social behavior is demonstrated when one option is selected by the population, while a scattering of individuals among the distinct options rather suggests responses to environmental stimuli (including competition for resources). In parallel, models taking into account social interactions and environmental preferences allow the identification of individual mechanisms that can generate the observed collective patterns (Jeanson et al., 2004b and Meunier et al., 2006).

In our study, we focus on one type of aggregation that is widely utilized by fishermen to enhance their catches: the aggregation of pelagic fish species under objects floating at the surface of the ocean (hereafter called FOBs). Thousands of individuals from several species can be found under a single piece of wood (such as a log) (Castro et al., 2002, Hunter and Mitchell, 1967 and Taquet et al., 2007b). Among these species, tropical tunas (skipjack - Katsuwonus pelamis, yellowfin - Thunnus albacares, bigeye $-T$. obesus) have received great attention in the last two decades, as more than half of the tropical tunas landed in the world by purse seine fisheries are caught when they are associated with FOB's (See ( Dagorn et al., 2013) for a review). The use of electronic tags (acoustic tags and archival tags) has allowed the investigation of the behavior of tunas in an array of FOBs ( Bach et al., 2003, Brill et al., 1999, Cayré, 1991, Cayré and Marsac, 1993, Dagorn et al., 2000a, Dagorn et al., 2007, Girard et al., 2004, Govinden et al., 2012, Holland et al., 1990, Klimley and Holloway, 1999, Marsac and Cayre, 1998, 
Mitsunaga et al. 2012; Ohta and Kakuma 2005; Robert et al. 2012; Schaefer and Fuller 2002, 2010; Taquet et al. 2007a), showing in particular that a tuna alternates between associative and unassociative phases (Dagorn et al. 2007; Govinden et al. 2012; Robert et al. 2012; Schaefer and Fuller 2010). Individual residence times around floating objects (associative phase), as well as the times between two consecutive associations with a FOB (unassociated phase), are highly variable, ranging from a few minutes up to several months (Dagorn et al. 2007; Ohta and Kakuma 2005; Mitsunaga et al. 2012). This variability is found at the individual level, as the same tuna can switch between short (few days) and long (few weeks) residence times depending on the local conditions around the FOB (e.g. prey availability, presence of conspecifics, abiotic conditions) (Robert et al. 2013a). The reasons why tuna associate with these structures and the underlying mechanisms are still poorly understood (see (Freon and Dagorn 2000) for a review). This behavior might rely on environmental cues, and one hypothesis often mentioned in the literature proposes that tuna use drifting objects to stay in contact with food-rich water masses. Indeed, natural floating objects are abundant in river mouths or concentrate in frontal zones offshore, two areas known for their high biological productivity (indicator log hypothesis - (Marsac et al. 2000)). A second hypothesis, the “meeting point hypothesis”, indicates that such surface heterogeneity could enhance the encounter rate between individuals or schools, allowing the formation of schools or increasing their sizes (Dagorn and Freon 1999; Freon and Dagorn 2000). In this scenario, floating objects would have an important role in the process of fusion between schools. Recent experiments support the existence of social interactions in the aggregation processes of fish with FOBs. The meeting point hypothesis has been experimentally validated for small pelagic species such as bigeye scad (Soria et al. 2009). In tuna, a first trial of a field-based binary choice experiment has highlighted the asymmetrical distribution of the population between two close and identical FOBs (Figure 1), suggesting the existence of social interactions (Robert et al. 2013b). In terms of the dynamics, this experiment has indicated an alternation of the winning FOB (the FOB that aggregated most of the biomass) over time. Temporal variation of the winning FOB in an array of FOBs has also been observed for other species that aggregate under FOBs (Capello et al. 2012). The departure of substantial numbers of tuna from a FOB has been observed by fishermen (Moreno et al. 2007), and acoustic tagging has highlighted synchronicity in the departure of several fish associated with the same floating object (Klimley and Holloway 1999; Dagorn et al. 2007; Govinden et al. 2012). Those observations suggest (but do not demonstrate) social synchronization in the departure process of fish from FOBs.

The objective of this work is to identify which mechanisms are most likely to reproduce the behavioral patterns of tuna aggregations around FOBs at both the individual (highly variable residence times and alternate of 
associated and unassociated phases) and the group levels (asymmetrical alternate distribution and synchronicity in departure), mentioned above (See Table S1 which summarized the information described above in the form of actual targets for the modeling outcomes). Several modeling approaches co-exist in the literature: from detailed descriptions of individual mechanisms to population level modeling, and from short (e.g. minutes) to long timescales (years). We developed a model at an intermediate time-scale (e.g. days and weeks) where the overall objective is to link individual behavior to collective patterns. Generally, in such cases, individuals are considered as "black boxes" with transition probabilities between different states where their behavior is modeled from a phenomenological point of view (e.g no emphasis on the underlying sensory systems of detection), with the objective of exploring the resulting emergent collective pattern.

We developed a theoretical approach where the mechanisms proposed in the literature to explain the aggregative behavior of tuna around FOBs are translated into phenomenological functions. We first applied a non social model, which was the common vision of tuna behavior around FADs (Hilborn and Medley 1989). Secondly, the application of a well-known retention model (model which has been mainly studied by entomologists (Ame et al. 2006; Jeanson and Deneubourg 2009) ) constitutes a first attempt to involve social interactions in the aggregative behavior of tuna around FOBs. The objective of the third model is to identify the condition to observe synchronism in departure from the FOBs. The synchronization pattern is a main characteristic of the meeting point hypothesis (Dagorn and Freon 1999; Freon and Dagorn 2000). Previous works, mainly on vertebrates, have focused on short time-scale modeling (second, minute) of the synchronization in departure observed in sheep and primates (Petit and Bon 2010). In such a short time-scale description, the departure is isolated from the global dynamics. Here we developed another phenomenological model of synchronous departures where the dynamics (departures and returns) are integrated into the global dynamics of the aggregations. Finally we studied a combination of two phenomenological functions (retention and synchronous departure) from which the resulting patterns are unknown.

Based on the simple case of a system of two FOBs, we studied the temporal dynamics and distribution of the population resulting from these different hypotheses on the social behavior of the animals (responses to conspecifics). Predictions of the models were qualitatively, and when possible quantitatively, compared to observations of tuna aggregations under FOBs at both the individual and the group levels. 


\section{Methods}

\section{Model description}

We use a system of differential equations and its stochastic counterpart to describe the evolution of the number of items (which could be individuals or small schools, e.g., see (Dagorn et al. 2000b) for a similar use of school units in models) under two FOBs through time. The models were studied using both differential equations and the corresponding stochastic simulations (Monte Carlo multi-agents), as complementary approaches.

\section{Ordinary differential equations}

We define two variables: $\mathrm{X}_{\mathrm{i}}$ is the population under $\mathrm{FOB}_{\mathrm{i}}\left(\right.$ with $\mathrm{i}=1$,2) and $\mathrm{X}_{\mathrm{e}}$ the population external to the FOBs (i.e., in the reservoir). In biological terms, the reservoir represents the open ocean environment. The model is based on two processes: joining and leaving a FOB, which are characterized by $\mathrm{R}_{\mathrm{i}}$, the rate of reaching $\mathrm{FOB}_{\mathrm{i}}$, and $\mathrm{Q}_{\mathrm{i}}$, the rate of leaving $\mathrm{FOB}_{\mathrm{i}}(\mathrm{Eq} 1 \mathrm{a}, \mathrm{b})$ (proportion of the items under a FOB leaving/reaching this FOB per unit of time). The system is closed, meaning that the total number of items $\left(\mathrm{N}=\mathrm{X}_{1}+\mathrm{X}_{2}+\mathrm{X}_{\mathrm{e}}\right)$ is conserved (i.e., we neglect recruitment and mortality - Eq 1b). The underlying assumption is that the movements between FOBs and the reservoir are much faster than recruitment and mortality (mean residence time under a FOB around a weak and in a network of FOBs around one to several months (Robert et al.2013a); whereas lifespan of tuna could archive 5-10 years) and that the recruitment of new fish in the population equals the mortality of fish. We assume that the population $\mathrm{X}_{\mathrm{e}}$ is homogeneously distributed within the environment and that one item cannot travel directly from one FOB to the other but must pass through the reservoir. All items are considered identical, and the two FOBs have the same characteristics. We assume that $\mathrm{R}_{\mathrm{i}}$, the rate of joining, is constant (and equal to $\mu / 2$ for each FOB, where $\mu$ is a kinetic constant, the simplest baseline assumption- see discussion section on improving model realism).

$$
\begin{aligned}
& \frac{d X_{i}}{d t}=R_{i} X_{e}-Q_{i} X_{i} \text { with } i=1,2 \text { (1a) } \\
& N=X_{e}+\sum_{i=1}^{2} X_{i}(1 \mathrm{~b})
\end{aligned}
$$

In this work, we propose four potential mechanisms based on local interactions that can drive the individual rate of leaving a FOB $\left(\mathrm{Q}_{\mathrm{i}}=\mathrm{f}\left(\mathrm{X}_{\mathrm{i}}\right)\right.$, Figure 2). Four models are tested: a non-social model with no interaction between items and three models with social interactions: a retention threshold model, a departure threshold model and a model combining retention and departure processes. 


\section{Non-social model}

The basic model describes the null hypothesis of the absence of social interactions in the leaving process $\left(Q_{i}=\right.$ constant, Eq 2), which has already been used in previous modeling of tuna aggregations around FOBs (Dagorn et al. 2000b; Hilborn and Medley 1989; Samples and Sproul 1985). In this model, the decision of an item to leave the $\mathrm{FOB}_{\mathrm{i}}$ is independent from the presence of conspecifics (Figure 2a). The presence of several items under a FOB is thus an epiphenomenon resulting from the sum of individual responses to an external signal, the presence of a FOB.

$Q i=\theta(2)$

\section{Retention model}

In a number of biological examples, aggregations result from retention forces between conspecifics (Ame et al. 2006; Sumpter and Pratt 2009; Halloy et al. 2007). As such, in this model, $Q_{i}$ decreases with an increasing number of items under $\mathrm{FOB}_{\mathrm{i}}(\mathrm{Eq} 3$, Figure $2 \mathrm{~b}) . \mathrm{\Theta} / \mathrm{K}^{\mathrm{n}}$ defines the maximal rate of leaving a $\mathrm{FOB}$, and $\mathrm{K}$ acts as a threshold. Biologically, n can be understood as the sensitivity of the social retention process to the population at the FOB. The non-social model (discussed above) is, in fact, the extreme case of $n=0$.

$$
Q i=\frac{\theta}{K^{n}+X_{i}^{n}}
$$

\section{Departure model}

The aim of this model is to reflect the meeting point hypothesis proposed in the literature (Dagorn and Freon 1999; Freon and Dagorn 2000). More precisely, this model displays synchronicity in departure, a phenomenon suggested by the acoustic tagging (Klimley and Holloway 1999; Dagorn et al. 2007; Govinden et al. 2012) and the collective departure of substantial amounts of aggregated biomass, as suggested by the knowledge of fishermen (Moreno et al. 2007). Several models of group departure have been developed by behavioral ecologists for several species and include various mechanisms from leadership to consensus decision making (e.g. for review (Couzin et al. 2005; Sumpter and Pratt 2009; Ward et al. 2008)). Often, the behavior of animals is splitted in two states (resting and leaving states) with the transition probability (from one state to the other) 
mainly depending on the population size in each behavioral state. As we are modeling the population dynamics on long time scales, we favored another approach in which the rate of leaving a FOB per unit of time is a threshold function. $\theta$ ' defines the maximal rate of leaving per unit of time, $n$ ' is the sensitivity of the individual interactions and $\mathrm{K}^{\prime}$ is the threshold number of items above which the rate of leaving increases rapidly (Eq 4,

Figure 2c). The departure of substantial amounts of the aggregated biomass occurs for n’ $\geq 2$.

$$
Q i=\frac{\theta^{\prime} X_{i}^{n^{\prime}}}{K^{n^{n^{\prime}}+X_{i}^{n^{\prime}}}}
$$

\section{Combination of retention and departure model}

The model in which social interactions arise as a linear combination of retention and collective departure (Eq 5) has been studied through simulation only, because the analytical resolution is impossible (especially for n'>2).

The resulting rate of leaving a FOB decreases with increasing $\mathrm{X}_{\mathrm{i}}$ until a threshold number of items aggregated under the FOB is reached (K'). The individual rate of leaving then increases sharply (Figure 2d).

$Q_{i}=\frac{\theta}{K^{n}+X_{i}^{n}}+\frac{\theta^{\prime} X_{i}^{n^{\prime}}}{K^{n^{\prime}}+X_{i}^{n^{\prime}}}$

\section{Stochastic simulations}

The initial conditions are set with all items in the reservoir $\left(X_{e}=N ; X_{1}=X_{2}=0\right)$. At each time step, the position of each item is checked. If an item is under $\mathrm{FOB}_{\mathrm{i}}$ at time $\mathrm{t}$, it can join the reservoir or stay under $\mathrm{FOB}_{\mathrm{i}}$. Its position at $\mathrm{t}+1$ depends on a comparison between the calculated probability of leaving $\mathrm{FOB}_{\mathrm{i}}$ and joining the reservoir $\left(\mathrm{Q}_{\mathrm{i}} \Delta \mathrm{t}_{\mathrm{i}}\right)$ and a random number sampled from a uniform distribution between 0 and 1 . If the value of the random number is less than the calculated probability $Q_{i}$, the item leaves $\mathrm{FOB}_{\mathrm{i}}$ and enters the reservoir. The probability $\mathrm{Q}_{\mathrm{i}}$ is updated at each time step according to the number of items under $\mathrm{FOB}_{\mathrm{i}}$. Similarly, items in the reservoir at time t can stay in the reservoir or join one of the two FOBs, with the probability of joining $\mathrm{FOB}_{\mathrm{i}}(\mathrm{Ri} \Delta \mathrm{t})$. The comparison between a random number and this probability is used to determine its position at time $t+1$. In our models, $R_{1}$ and $R_{2}$ are equal and constant. Depending on the model, the probability of leaving is constant (non-social model), decreases with the population around the FOB (retention model), increases with this population (departure model) or shows a minimum (retention-departure model). The simulations are run for different total numbers $\mathrm{N}$ of items, N=10, 50 and 500. Each condition was run 500 times for 5000 time steps to reach a stationary state. A preliminary analysis has shown that much longer simulations showed similar patterns than those presented below. 


\section{Analysis of the models}

Differential equations are good predictors of the main properties of the systems. For example, steady states provide reference points on the resulting distributions of populations and on the most probable states of the systems. Steady states and their stability were analyzed using linear stability analysis and numerical resolution. However, the use of differential equations remains questionable when $\mathrm{N}$ is small.

Stochastic simulations were performed as the primary approach allowing the introduction of stochasticity in decision rules and the calculation of item-based metrics, such as residence times under FOBs. The dynamic of the systems through time was studied using stochastic simulations to account for the randomness of the processes and also because the solution of differential equations only depends on the initial conditions.

\section{Model parameterization and comparison to observed patterns}

We compared the outputs of our models with existing data or knowledge. Following results from a binary choice experiment (see (Robert et al. 2013b) for detailed description of experimental design and data analysis), our objective was to find a model that could generate both asymmetrical and symmetrical distributions of populations between two identical FOBs in a homogeneous environment (Figure 1). We highlighted that the symmetrical distribution of the number of items between two identical FOBs (dispersal solution $\mathrm{X}_{1}=\mathrm{X}_{2}$ ) is a solution of all models, including those relying on social interactions. However, the reverse (i.e., the selection of one FOB) is only possible if social interactions are incorporated into the model. These results show that it is not sufficient to consider only the final result and emphasize the need to also consider the stochasticity and the dynamics of the system. As such, it is not convenient to use the solutions of the differential equations because they give mean results. Instead, the use of stochastic simulations allows comparing the evolution of the number of items under the FOBs, as well as the frequency of the distribution of populations between the two FOBs $\left(\mathrm{X}_{1} /\left(\mathrm{X}_{1}+\mathrm{X}_{2}\right)\right)$, with observations of tuna aggregative behavior around FOBs. The last time step of the 500 simulations derived for a given parameter set was used to generate the frequency of the distribution of populations between the two FOBs $\left(\mathrm{X}_{1} /\left(\mathrm{X}_{1}+\mathrm{X}_{2}\right)\right)$. We performed a Kolmogorov test to compare experimental distributions derived from a binary choice experiment (Robert et al. 2013b) with simulation-based histograms. 


\section{Results}

Analysis of the models

The non-social model and the retention model are well described and studied in the literature (Ame et al. 2006; Halloy et al. 2007; Sumpter and Pratt 2003, 2009). The third model proposed here (the departure model) shares the same objective than other published models but using a different formalism and scale (Petit et al. 2009; Sueur et al. 2011). Main properties of these models are presented, keeping in mind that the last model we studied is a combination of both retention and departure processes.

\section{Non-social model}

This system has only one stable steady state (Eq 6), in which the number of items under one FOB is the same as the number of items under the other FOB. The ratio $\Theta / \mu$ influences the fraction of the population under the FOBs $\left(\left(\mathrm{X}_{1}+\mathrm{X}_{2}\right) / \mathrm{N}\right)$. One basic property of this linear model is that residence times under the FOBs and in the reservoir are exponentially distributed and independent of the aggregated population.

$$
X_{1}=X_{2}=\frac{N}{2\left(1+\frac{\theta}{\mu}\right)}
$$

For a ratio $\Theta / \mu=1$, the mean residence times under both FOBs and in the reservoir are equal. This result is consistent with a duration of the associated phases that is of the same order of magnitude as the duration of the unassociated phases (Dagorn et al. 2007). Not surprisingly, simulations confirm that, on average, the fraction of the population under the FOBs $\left(\left(\mathrm{X}_{1}+\mathrm{X}_{2}\right) / \mathrm{N}\right)$ is 0.5 (Figure $\left.3 \mathrm{a}, \mathrm{b}\right)$. This result is unchanged if $\mathrm{N}$ is increased (Figure S1).

\section{Retention model}

For $n=1$, the model has only one stable steady state, characterized by an equal number of items in both sites (Eq 7).

$$
k^{n}\left(X_{1}-X_{2}\right)-X_{1} X_{2}\left(X_{1}^{n-1}-X_{2}^{n-1}\right)=0
$$

For $n=2$, the model may have two families of real and positive stationary solutions: 1 or 3 symmetrical stationary states and 0, 2 or 4 asymmetrical (Figure S3). Again, the first solutions (dispersal) are an equal distribution of the 
population under both FOBs $\left(\mathrm{X}_{1}=\mathrm{X}_{2}\right)$. The other solutions are asymmetrical, with unequal numbers of items at each site $\left(\mathrm{X}_{1}>\mathrm{X}_{2} ; \mathrm{X}_{1}<\mathrm{X}_{2}\right)$. The real and positive solutions are presented in the bifurcation diagram (Figure 4) of $\mathrm{X}_{1} / \mathrm{N}$ (or $\mathrm{X}_{2} / \mathrm{N}$ ) as a function of $\mathrm{N}$. For the parameter sets used to construct the figure, subcritical bifurcation occurs at approximately $\mathrm{N}=8$. For larger populations, the dispersal state $\left(\mathrm{X}_{1}=\mathrm{X}_{2}\right)$ is unstable, and one of the two asymmetrical solutions is selected. At the stationary solution, the number of items under the FOBs is almost constant, and the residence times are exponentially distributed with a mean residence time equal to $\left(\mathrm{K}^{\mathrm{n}}+\mathrm{X}_{\mathrm{i}}^{\mathrm{n}}\right) / \Theta$. The fraction of the total population under the FOBs $\left(\left(X_{1}+X_{2}\right) / N\right)$ varies as a function of $\Theta / \mu$.

The simulations provide supplementary information. Although the deterministic equations predict the selection of one site for $\mathrm{N}=10$, the number of items under the FOBs is too small (2, on average) in the simulations to stabilize the selection of one FOB (Figure S2 a,b). If $\mathrm{N}$ is increased ( $\mathrm{N}=50)$, the number of items under one FOB, via the retention process, induces the gathering of the entire population under one $\mathrm{FOB}\left(\left(\mathrm{X}_{1}+\mathrm{X}_{2}\right) / \mathrm{N}=0.97\right.$, with $\mathrm{X}_{1}>>\mathrm{X}_{2}$ or $\mathrm{X}_{1}<<\mathrm{X}_{2}$ ) and so until the end of the simulation (Figure 3d). Each simulation ends with a clear winning FOB, each FOB having a 50\% probability of winning (Figure 3c). This selection occurs through amplification in the sense that due to positive interactions, a small change in the population around a FOB is amplified time step after time step. Nevertheless, if $\mathrm{N}$ is large $(\mathrm{N}=500)$, the number of items under each site rapidly attains a high value. In this situation, the resulting probability of leaving is very low and maintains the population under each FOB (Figure S2f). As such, the time to reach the selection can be very long (not observed in the simulations) and may be meaningless at the biological scale of tuna aggregations under FOBs. The simulations are therefore characterized by a symmetrical distribution of the entire population under both FOBs (Figure S2c).

\section{Departure model}

The differential equation model has only one positive real and stable solution for all values of n', characterized by a symmetrical distribution of the population under each FOB (Eq 8).

$$
K^{\prime n^{\prime}}\left(X_{1}^{n^{\prime}-1}-X_{2}^{n^{\prime}-1}\right)+X_{1}^{n^{\prime}} X_{2}^{n^{\prime}}\left(X_{1}-X_{2}\right)=0
$$

For high values of n', the individual response to the threshold is quasi-deterministic (Figure 2c). Simulations were run with n'=100. The sensitivity analysis derived for parameter $K^{\prime}$ illustrates how the ratio between N/K' influences the degree of dependency between FOBs (Figure S4). Simulations in which $\mathrm{K}$ ' $<\mathrm{N} / 2$ show that each FOB accumulates items until the threshold number of items is reached; at this point, the resulting probability of 
leaving changes from a value close to 0 to a value close to 1 , producing a substantial number of simultaneous departures (Figure 3f). Most important, although the model generates significant fluctuations of population under the FOBs through time, the number of items under both FOBs is identical on average $\left(\mathrm{X}_{1} /\left(\mathrm{X}_{1}+\mathrm{X}_{2}\right)=0.5\right.$, Figure 3e).

For $\mathrm{K}^{\prime}>\mathrm{N} / 2$, the threshold number of items cannot be achieved under the FOBs. The resulting probability of leaving is maintained at a very low level. Simulations illustrate how the entire population distributes equally between the two FOBs (Figure S4e,f).

The simulations indicate that the mean residence time decreases with increasing $\mathrm{N}$ and $\mu$ and increases with increasing $K$. The model properties and behavior described above $\left(K^{\prime}>N / 2\right.$ or $\left.K^{\prime}<N / 2\right)$ are robust to different values of $\mathrm{N}$.

Combination of retention and departure model

The model sensitivity to the parameters was investigated for $\mathrm{N}=50$. In a configuration where the two FOBs compete $\left(K^{\prime}>N / 2\right)$, both processes drive the population dynamics. The retention process allows the maintenance of large aggregations under one FOB through time until the departure threshold is reached, resulting in a significant reduction of the number of aggregated items (Figure 3h). For these parameter sets, the winning FOB alternates with time. The time during which the aggregation is maintained, the frequency of collective departure and the fraction of the total population under the FOBs $\left(\left(\mathrm{X}_{1}+\mathrm{X}_{2}\right) / \mathrm{N}\right)$ varies as a function of $\Theta^{\prime} / \mu$. If $\mathrm{K}^{\prime}<\mathrm{N} / 2$, simulations indicate that the departure process alone is driving the dynamics (Figure S5e,f). For N=10, the simulations suggest that the number of items under a FOB is never sufficiently large to initiate the retention process (Figure S5a,b). The dynamic is therefore similar to what is observed for the retention model for small N. For $\mathrm{N}=500$, the number of items under the FOBs rapidly attains a high value. The resulting probability of leaving a FOB is therefore very low and maintains a quasi-constant population under the FOBs $\left(\left(\mathrm{X}_{1}+\mathrm{X}_{2}\right) / \mathrm{N}=0.99\right.$, with $\mathrm{X}_{1} \approx \mathrm{X}_{2}$; Figure S5g,h). As such, the time to reach the threshold can be very long (not observed in the simulations) and may be meaningless at the biological scale of tuna aggregation under FOBs. The dynamic is similar to that observed for the retention model for large N. 
For the range of parameters tested, the interplay between retention and collective departure processes drives the dynamic of the system only if $\mathrm{K}>\mathrm{N} / 2$ and large $\mathrm{N}(\mathrm{N}=50)$.

\section{Model parameterization and comparison to observed patterns}

One important and common feature of all models presented here is that the standard deviation of the histograms varies with the number of items (see supplementary). We limited the detail comparison of simulations displayed with $\mathrm{N}=50$ with the joined distribution of both setups of the binary choice experiment (Robert et al. 2013b).

While this choice is rather arbitrary, it can be seen as the corollary of the hypothesis of considering that one unit in our model corresponds to a tuna school of one ton, which allows us to compare our theoretical results with experimental data expressed in tons (Figure 1). Nevertheless, full comparisons with other population sizes (N) are reported in the supplementary (Table S2) and implications of varying $\mathrm{N}$ are extensively discussed later on. Comparison of modeling targets and model outputs are provided in Table S1.

Obviously the non-social model cannot generate asymmetrical distributions of a population in a homogeneous environment, whatever the value of $\mathrm{N}$. The emergence of a clear winning FOB is an outcome of the retention model for certain parameter values, the necessary condition being $n>1$. In this situation of a clear winning FOB, the residence times of items under the FOBs are quasi-infinite (equal to the duration of the simulation), a prediction that does not agree with observations collected in the wild (Dagorn et al. 2007; Schaefer and Fuller 2010). Moreover, the simulations generate bimodal distributions of the fraction of the total population under the FOBs $(\mathrm{X} 1 /(\mathrm{X} 1+\mathrm{X} 2))$ on 0 and 1, extreme patterns not observed in the binary choice experiment. For $\mathrm{N}=50$, the experimental distribution is statistically different from the simulation-based distribution (p-value $=0.0001)$. Nonetheless, it is not excluded that certain parameter values could smooth the asymmetry. This model can also generate a symmetrical distribution in the case of very low numbers of items in the simulation.

The departure model generates large fluctuations of the aggregated number of items under the FOBs, as well as a collective departure of a substantial fraction of the aggregated population, which can lead to temporal desynchronization between the two FOBs. However, on average, the population is equally distributed between the two FOBs. The experimental distribution could be generated by such behavioral rules ( $\mathrm{p}$ value=0.24). Nevertheless, the individual residence times, with an average equal the number of time steps required to reach the threshold, have a relatively low standard deviation. This low standard deviation is not fully consistent with the high intra individual variability observed in the residence times of tuna under FOBs (Dagorn et al. 2007; Ohta and Kakuma 2005; Robert et al. 2013a; Schaefer and Fuller 2010). For certain parameter values, 
simulations based on the linear combination of the retention and departure processes generate an alternation of the winning FOB through time, the necessary condition being $N>K^{\prime}>N / 2$. The retention process engenders and maintains the aggregation, whereas the threshold departure function breaks the aggregation. This scenario is consistent with the general knowledge of tuna aggregation dynamics under FOBs (Table S1 and S2), at least qualitatively (except if $\mathrm{N}=500$ ). Moreover, the distribution resulting from the binary choice experiment can be generated by such behavioral rules ( $\mathrm{p}$ value $=0.39$ if $\mathrm{N}=50$ ). This model allows the maintenance of the aggregation over a longer time period than the threshold departure model. This result is more likely to reproduce the wide range of residence times observed in the wild (in particular, the long residence times of several weeks

displayed by some tagged tuna at FOBs (Dagorn et al. 2007)). Furthermore, it also generates extended periods of time during which a FOB can be considered "empty”. This pattern, observed in the field, is not reproduced by the threshold departure model.

\section{Discussion}

Using behavioral models, and through the comparison of the outputs of the simulations with knowledge on the behavior of tuna around FOBs, we aimed at identifying which mechanisms are most likely to reproduce the general characteristics of tuna aggregations under FOBs at both the individual and the group levels. The characterization of these mechanisms is (i) an important step towards our understanding of the associative behavior of tunas with FOBs and (ii) a priority for science-based fishery management. This work also provides new insights into the dynamics and distribution patterns of populations in an environment with aggregating sites, following different hypotheses on the individual's responses to conspecifics when the number of items (individuals or group/schools) increases (N). We also provide new insights on the population dynamics and distributions that could result from a combination of behavioral rules (retention and collective departure).

One important result of this study is that the patterns of tuna aggregations around FOBs, at both the individual and group levels, cannot be generated by non-social systems. This result reinforces the recent conclusion of a binary choice experiment (Robert et al. 2013b) and other studies on small pelagic fish (Capello et al. 2011; Soria et al. 2009) on the role of social behavior in fish aggregations, which was mainly ignored in the past. Since the early 1990’s, tropical tuna purse seine vessels have been exploiting the aggregative behavior of tuna around FOBs by deploying man-made floating objects (often called FADs -Fish Aggregating Devices). The deployment of thousands of FADs in the tropical ocean has increased the catchability of tuna. Currently, 40\% of the global tuna catch results from fishing operations by purse seine vessels performed around artificial or 
natural FOBs. The use of this new fishing tool has raised concerns for the sustainability of this mode of fishing and about the ecological consequences of the deployment of FADs in the ocean (Dagorn et al. 2013; Fonteneau et al. 2000; Miyake et al. 2010). The increasing density of FOBs could increase the residence times of tuna in one area and/or modify their migration patterns, which in turn could negatively influence their condition (e.g., survival, reproduction) as expressed in the ecological trap hypothesis (Hallier and Gaertner 2008; Marsac et al. 2000). Results from different studies have provided contrasting results, preventing any conclusive evaluation of this hypothesis (see review in Dagorn et al. 2013). No study, however, has considered the possible interplay between social behavior and FOBs in the determination of the residence time of tuna in one area. The probability functions in the three social models presented herein share the common property of quorum responses to conspecifics, in which an animal's probability of committing to a particular option increases sharply if a threshold number of other individuals have committed to it. This important mechanism of decision-making in animals has previously been proposed for fish (Sumpter and Pratt 2009). Such mechanisms could lead to well-known hysteresis effects (Beckers et al. 1992; Beekman et al. 2001). Applied to our case study, if an aggregation is formed under a FOB located in a food-rich area and the FOB then drifts to a foodpoor area, social retention processes could delay the departure of the fish from the FOB (an outcome that would not occur for non-social species) if social motivation is stronger than feeding motivation. In an extreme case, in a scenario with a high density of FOBs and a low population size, the number of fish under the FOBs would never be sufficiently large to initiate a collective departure from the FOBs. To assess the impacts of FADs on the ecology of tuna, it is necessary to determine whether the social behavior of tuna plays a role in the aggregations they form under FOBs, and, if so, how social behavior contributes to this role.

Unfortunately, the little amount of empirical data available so far is not sufficient to fully validate the model. One valuable output of the theoretical approach is to help to define the key parameters that future experimental work should emphasize. Although the model scenario proposed here constitutes a starting point, progress should be done in the model parameterization and validation. Simultaneous measurements of individual residence times and number of tuna aggregated around the same FOB would be necessary to guide the choice of the most appropriate form of the probability to leave a FOB. To complete the validation, further and longer binary choice experiments, combined with individual tagging, is recommended. Furthermore, fishermen are routinely using echo-sounder buoys to remotely monitor (using GPS technology) the amount of aggregated tuna under FOBs, which currently constitutes the only available way to access large datasets of tuna populations distributed within an array of FOBs. 
There is no doubt that our study suffers from some weaknesses. Our analyses focus on a restricted number of FOBs in a homogeneous environment and a constant population. Inferring model properties when these three variables may vary simultaneously is far from trivial. Interestingly, for a given number of aggregating sites (FOBs e.g herein 2) we illustrated how model properties and behaviors vary when $\mathrm{N}$, the number of items in the simulation, is increasing. This behavior is a common property of density-dependent systems (Camazine et al. 2001; Sumpter 2010; Astudillo Fernandez et al. 2012). Group-level properties such as animal aggregations are the result of movements of individuals or groups of individuals. Although tuna are known to school, little is known about the schooling behavior of tuna around floating objects and the influence of these schooling dynamics in the formation, maintenance and dispersion of aggregations (Robert et al. 2013b). As expressed earlier, the items can be considered as schools or individual fish. Therefore, a given biomass (expressed in tons) will generate distinct patterns depending on the number and the size of schools that join and leave the aggregating sites (FOBs). Another related prediction would be that for a given $\mathrm{N}$ (local population or number of schools) the spatial and temporal characteristics of the aggregation may vary when the number of aggregating sites is increasing. As a consequence, one important result of this study is that without a detailed description of the number of FOBs and the number of tuna schools that reach and leave the FOBs, it would be difficult to unequivocally identify the underlying mechanisms involved in these aggregations. To go further, a more realistic approach would then require modeling the social interactions between fish when they are not associated with FOBs, mainly the probability of fusion and fission between free swimming schools.

Some models properties remain valid when the number of FOBs is increasing in a heterogeneous environment (with a constant population). For the non-social model, the proportion of the population associated with each FOB will be proportional to their respective quality and the fraction of the entire population associated with FOBs will increase with their number (Hilborn and Medley 1989). For the retention model, the resulting spatial patterns will depend on the relative forces between the intrinsic retention power of the FOBs (their quality) and the strength of the social interactions (Sempo et al. 2013). A preliminary analysis indicates that the relative value of the total population and the number of FOBs, as well as the initial conditions, will determine the degree of competition between the FOBs and therefore the resulting distribution patterns (selection of some FOBs or scattering of the population among all FOBs). For the departure model and the one combining both retention and departure processes, the resulting temporal dynamics and population distributions are not trivial and deserve dedicated analyses. 
Important questions remain. How could tuna assess the number of conspecifics under a FOB? Does this mechanism rely on direct or indirect cues? The sensory system often mentioned is hearing because the range of perception of sound in water is higher than vision and a role for chemical cues is not supported by certain experiments (Dempster and Kingsford 2003). The sounds made by tuna and other species in aggregations may be the type of information used by tuna to assess the amount of biomass aggregated (Girard et al. 2004). Our models can be adapted to incorporate intra and interspecific interactions in the process of attraction to FOBs. The modeling parameter $\mathrm{R}_{\mathrm{i}}$ could be for example a function of the entire associated biomass (multispecific aggregation). Such a model would share the same properties described for the retention model, at least qualitatively (primarily, the generation of asymmetrical distributions of individuals if $n>1$ ). Indeed, although the retention of individuals leads to an increase in local population density, the inflow of individuals can essentially produce large aggregations despite short residence times. General properties of models including retention or attraction, or both, are similar (Nicolis and Deneubourg 1999, Sempo et al. 2013). Additionally, the individual decision to leave a group is often presented as a balance between individual preferences (e.g., environmental conditions and physiological stages) and the advantage of remaining a member of the group (Conradt and Roper 2000, 2009). The same reasoning can be applied to the decision to leave a specific site (e.g., a FOB), if several conspecifics are present. This trade-off has been extensively investigated for schooling and shoaling fish. However, to date no analysis has been performed to examine how the physiological state (e.g., starvation, reproductive status) of the fish affects their individual responses to FOBs and congeners.

\section{Acknowledgments}

This work was carried out with financial support from the Commission of the European Communities, specifically the RTD programme of Framework Programme 7, “Theme 2-Food, Agriculture, Fisheries and Biotechnology,'” through the research project, MADE (mitigating adverse ecological impacts of open ocean fisheries) and by the Alice and David Van Burren Foundation. This report does not necessarily reflect the views of the Commission and in no way anticipates the Commission's future policy in this area. J.L.D. is a senior research associate from the Belgian National Fund for Scientific Research (F.N.R.S). This work was also supported by an Action de Recherche Concertée de la Communauté française de Belgique: Individual and collective issues in dispersal and aggregation: from proximal causes to ultimate consequences at contrasting scales. 


\section{References}

Ame JM, Halloy J, Rivault C, Detrain C, Deneubourg JL (2006) Collegial decision making based on social amplification leads to optimal group formation. Proceedings of the National Academy of Sciences of the United States of America 103 (15):5835-5840. doi:10.1073/pnas.0507877103

Astudillo Fernandez A, Hance T, Deneubourg JL (2012) Interplay between Allee effects and collective movement in metapopulations. Oikos 121 (6):813-822. doi:10.1111/j.16000706.2011.20181.x

Bach P, Dagorn L, Bertranda A, Josse E, Misselis C (2003) Acoustic telemetry versus monitored longline fishing for studying the vertical distribution of pelagic fish: bigeye tuna (Thunnus obesus) in French Polynesia. Fisheries Research 60 (2-3):PII S0165-7836(0102)00180-00187

Beckers R, Deneubourg JL, Goss S (1992) Trails and U-Turns in the selection of path by ant Lasius Niger. Journal of Theoretical Biology 159 (4):397-415. doi:10.1016/s0022-5193(05)80686-1

Beekman M, Sumpter DJT, Ratnieks FLW (2001) Phase transition between disordered and ordered foraging in Pharaoh's ants. Proceedings of the National Academy of Sciences of the United States of America 98 (17):9703-9706. doi:10.1073/pnas.161285298

Brill RW, Block BA, Boggs CH, Bigelow KA, Freund EV, Marcinek DJ (1999) Horizontal movements and depth distribution of large adult yellowfin tuna (Thunnus albacares) near the Hawaiian Islands, recorded using ultrasonic telemetry: implications for the physiological ecology of pelagic fishes. Marine Biology 133 (3):395-408

Camazine S, Deneubourg J, Franks N, Sneyd J, Theraulaz G, al e (2001) Self-Organization in Biological Systems. Princeton Univ. Press., Princeton

Canonge S, Deneubourg JL, Sempo G (2011) Group living enhances individual resources discrimination: The use of public information by cockroaches to assess shelter quality. Plos One 6 (6). doi:e1974810.1371/journal.pone.0019748

Capello M, Soria M, Cotel P, Deneubourg JL, Dagorn L (2011) Quantifying the Interplay between Environmental and Social Effects on Aggregated-Fish Dynamics. Plos One 6 (12). doi:e2810910.1371/journal.pone.0028109

Capello M, Soria M, Cotel P, Potin G, Dagorn L, Freon P (2012) The heterogeneous spatial and temporal patterns of behavior of small pelagic fish in an array of Fish Aggregating Devices (FADs). Journal of Experimental Marine Biology and Ecology 430:56-62. doi:10.1016/j.jembe.2012.06.022

Castro JJ, Santiago JA, Santana-Ortega AT (2002) A general theory on fish aggregation to floating objects: An alternative to the meeting point hypothesis. Reviews in Fish Biology and Fisheries $11(3): 255-277$

Cayré P (1991) Behaviour of yellowfin tuna (Thunnus albacares) and skipjack tuna (Katsuwonus pelamis) around fish aggregating devices (FADs) in the Comoros Islands as determined by ultrasonic tagging. Aquat Living Resources 4:1-12

Cayré P, Marsac F (1993) Modelling the yellowfin tuna (Thunnus albacares) vertical distribution using sonic tagging results and local environmental parameters. . Aquat Living Resources 6:1-14

Conradt L, Roper TJ (2000) Activity synchrony and social cohesion: a fission-fusion model. Proceedings of the Royal Society of London Series B-Biological Sciences 267 (1458):22132218

Conradt L, Roper TJ (2009) Conflicts of interest and the evolution of decision sharing. Philosophical Transactions of the Royal Society B-Biological Sciences 364 (1518):807-819. doi:10.1098/rstb.2008.0257

Couzin ID, Krause J, Franks NR, Levin SA (2005) Effective leadership and decision-making in animal groups on the move. Nature 433 (7025):513-516. doi:10.1038/nature03236

Dagorn L, Bach P, Josse E (2000a) Movement patterns of large bigeye tuna (Thunnus obesus) in the open ocean, determined using ultrasonic telemetry. Marine Biology 136 (2):361-371 
Dagorn L, Freon P (1999) Tropical tuna associated with floating objects: a simulation study of the meeting point hypothesis. Canadian Journal of Fisheries and Aquatic Sciences 56 (6):984-993

Dagorn L, Holland K, Restrepo V, Moreno G (2013) Is it good or bad to fish with FADs ? What are the real impacts of the use of drifting FADs on pelagic marine ecosystems ? . Fish and Fisheries In press. doi:DOI: 10.1111/j.1467-2979.2012.00478.x

Dagorn L, Holland KN, Itano DG (2007) Behavior of yellowfin (Thunnus albacares) and bigeye (Tobesus) tuna in a network of fish aggregating devices (FADs). Marine Biology 151 (2):595-606. doi:10.1007/s00227-006-0511-1

Dagorn L, Josse E, Bach P, Bertrand A (2000b) Modeling tuna behaviour near floating objects: from individuals to aggregations. Aquatic Living Resources 13 (4):203-211

Dempster T, Kingsford MJ (2003) Homing of pelagic fish to fish aggregation devices (FADs): the role of sensory cues. Marine Ecology-Progress Series 258:213-222

Dussutour A, Fourcassie V, Helbing D, Deneubourg JL (2004) Optimal traffic organization in ants under crowded conditions. Nature 428 (6978):70-73. doi:10.1038/nature02344

Fonteneau A, Pallares P, Pianet R (2000) Worldwide review of purse-seine fisheries on FADs. Paper presented at the Pêche thonière et dispositifs de concentration de poissons Martinique

Freon P, Dagorn L (2000) Review of fish associative behaviour: toward a generalisation of the meeting point hypothesis. Reviews in Fish Biology and Fisheries 10 (2):183-207

Girard C, Benhamou S, Dagorn L (2004) FAD: Fish Aggregating Device or Fish Attracting Device? A new analysis of yellowfin tuna movements around floating objects. Animal Behaviour 67:319326. doi:10.1016/j.anbehav.2003.07.007

Govinden R, Jauhary R, Filmalter J, Forget F, Soria M, Adam S, Dagorn L (2012) Movement behaviour of skipjack (Katsuwonus pelamis) and yellowfin (Thunnus albacares) tuna at anchored fish aggregating devices (FADs) in the Maldives investigated using acoustic telemetry. Aquatic Living Resources 25 (In press)

Hallier JP, Gaertner D (2008) Drifting fish aggregation devices could act as an ecological trap for tropical tuna species. Marine Ecology-Progress Series 353:255-264. doi:10.3354/meps07180

Halloy J, Sempo G, Caprari G, Rivault C, Asadpour M, Tache F, Said I, Durier V, Canonge S, Ame JM, Detrain C, Correll N, Martinoli A, Mondada F, Siegwart R, Deneubourg JL (2007) Social integration of robots into groups of cockroaches to control self-organized choices. Science 318 (5853):1155-1158. doi:10.1126/science.1144259

Hilborn R, Medley P (1989) Tuna Purse-Seine Fishing with Fish-Aggregating Devices (Fad) - Models of Tuna Fad Interactions. Canadian Journal of Fisheries and Aquatic Sciences 46 (1):28-32. doi:10.1139/f89-004

Holland KN, Brill RW, Chang RKC (1990) Horizontal and vertical movements of yellowfin and bigeye tuna associated with Fish Aggregating Devices. Fishery Bulletin 88 (3):493-507

Hunter JR, Mitchell CT (1967) Association of fishes with flotsam in the offshore waters of central America. Fish Bull US 66:13-29. doi:10.1093/icesjms/31.3.427

Jeanson R, Deneubourg JL (2009) Positive feedback, convergent collective patterns and social transitions in Arthropods Genome to social complexity

Jeanson R, Deneubourg JL, Grimal A, Theraulaz G (2004a) Modulation of individual behavior and collective decision-making during aggregation site selection by the ant Messor barbarus. Behavioral Ecology and Sociobiology 55 (4):388-394. doi:10.1007/s00265-003-0716-y

Jeanson R, Deneubourg JL, Theraulaz G (2004b) Discrete dragline attachment induces aggregation in spiderlings of a solitary species. Animal Behaviour 67:531-537. doi:10.1016/j.anbehav.2003.06.013

Klimley AP, Holloway CF (1999) School fidelity and homing synchronicity of yellowfin tuna, Thunnus albacares. Marine Biology 133 (2):307-317. doi:10.1007/s002270050469

Krause J, Ruxton GD (2002) Living in Groups Oxford Univ. Press, Oxford

Marsac F, Cayre P (1998) Telemetry applied to behaviour analysis of yellowfin tuna (Thunnus albacares, Bonnaterre, 1788) movements in a network of fish aggregating devices. Hydrobiologia 372:155-171 
Marsac F, Fonteneau A, Ménard F (2000) Drifting FADs used in tuna fisheries: an ecological trap? Paper presented at the Pêche thonière et dispositifs de concentration de poissons, Martinique

Meunier H, Leca JB, Deneubourg JL, Petit O (2006) Group movement decisions in capuchin monkeys: the utility of an experimental study and a mathematical model to explore the relationship between individual and collective behaviours. Behaviour 143:1511-1527

Mitsunaga Y, Endo C, Anraku K, Selorio CM, Babaran RP (2012) Association of early juvenile yellowfin tuna Thunnus albacares with a network of payaos in the Philippines. Fisheries Science 78 (1):15-22. doi:10.1007/s12562-011-0431-y

Miyake M, Guillotreau P, Sun CH, Ishimura G (2010) Recent developments in the tuna industry: stocks, fisheries, management, processing, trade and markets. vol 543. Rome

Moreno G, Dagorn L, Sancho G, Itano D (2007) Fish behaviour from fishers' knowledge: the case study of tropical tuna around drifting fish aggregating devices (DFADs). Canadian Journal of Fisheries and Aquatic Sciences 64 (11):1517-1528. doi:10.1139/f07-113

Nicolis SC, Deneubourg JL (1999) Emerging patterns and food recruitment in ants: an analytical study. Journal of Theoretical Biology 198 (4):575-592. doi:10.1006/jtbi.1999.0934

Ohta I, Kakuma S (2005) Periodic behavior and residence time of yellowfin and bigeye tuna associated with fish aggregating devices around Okinawa Islands, as identified with automated listening stations. Marine Biology 146 (3):581-594. doi:10.1007/s00227-004$1456-x$

Parrish JK, Edelstein-Keshet L (1999) Complexity, pattern, and evolutionary trade-offs in animal aggregation. Science 284 (5411):99-101. doi:10.1126/science.284.5411.99

Petit O, Bon R (2010) Decision-making processes: The case of collective movements. Behavioural Processes 84 (3):635-647. doi:10.1016/j.beproc.2010.04.009

Petit O, Gautrais J, Leca JB, Theraulaz G, Deneubourg JL (2009) Collective decision-making in whitefaced capuchin monkeys. Proceedings of the Royal Society B-Biological Sciences 276 (1672):3495-3503. doi:10.1098/rspb.2009.0983

Robert M, Dagorn L, Deneubourg JL, Itano D, Holland K (2012) Size-dependent behavior of tuna in an array of fish aggregating devices (FADs). Marine Biology 159 (4):907-914. doi:10.1007/s00227-011-1868-3

Robert M, Dagorn L, Filmalter J, Deneubourg JL, Itano D, Holland K (2013a) Intra-individual behavioral variability displayed by tuna at fish aggregating devices (FADs). Marine Ecology Progress Series DOI:10.3354/meps10303

Robert M, Dagorn L, Lopez J, Moreno G, Deneubourg JL (2013b) Does social behavior influence the dynamics of aggregations formed by tropical tunas around floating objects? An experimental approach. Journal of Experimental Marine Biology and Ecology (440):238-243

Samples KC, Sproul JT (1985) Fish aggregating devices and open-access commercial fisheries - A theoretical inquiry Bulletin of Marine Science 37 (1):305-317

Schaefer KM, Fuller DW (2002) Movements, behavior, and habitat selection of bigeye tuna (Thunnus obesus) in the eastern equatorial Pacific, ascertained through archival tags. Fishery Bulletin 100 (4):765-788

Schaefer KM, Fuller DW (2010) Vertical movements, behavior, and habitat of bigeye tuna (Thunnus obesus) in the equatorial eastern Pacific Ocean, ascertained from archival tag data. Marine Biology 157 (12):2625-2642. doi:10.1007/s00227-010-1524-3

Sempo G, Dagorn L, Robert M, Deneubourg J (2013) Impact of increasing deployment of artificial floating objects on the spatial distribution of social fish species. Journal of Applied Ecology In press

Soria M, Dagorn L, Potin G, Freon P (2009) First field-based experiment supporting the meeting point hypothesis for schooling in pelagic fish. Animal Behaviour 78 (6):1441-1446.

doi:10.1016/j.anbehav.2009.09.025 
Sueur C, Deneubourg JL, Petit O (2011) From the first intention movement to the last joiner: macaques combine mimetic rules to optimize their collective decisions. Proceedings of the Royal Society B-Biological Sciences 278 (1712):1697-1704. doi:10.1098/rspb.2010.2084

Sumpter D (2010) Collective Animal Behavior Princeton University Press,

Sumpter DJT, Pratt SC (2003) A modelling framework for understanding social insect foraging. Behavioral Ecology and Sociobiology 53 (3):131-144. doi:10.1007/s00265-002-0549-0

Sumpter DJT, Pratt SC (2009) Quorum responses and consensus decision making. Philosophical Transactions of the Royal Society B-Biological Sciences 364 (1518):743-753. doi:10.1098/rstb.2008.0204

Taquet M, Dagorn L, Gaertner JC, Girard C, Aumerruddy R, Sancho G, Itano D (2007a) Behavior of dolphinfish (Coryphaena hippurus) around drifting FADs as observed from automated acoustic receivers. Aquatic Living Resources 20 (4):323-330. doi:10.1051/alr:2008008

Taquet M, Sancho G, Dagorn L, Gaertner JC, Itano D, Aumeeruddy R, Wendling B, Peignon C (2007b) Characterizing fish communities associated with drifting fish aggregating devices (FADs) in the Western Indian Ocean using underwater visual surveys. Aquatic Living Resources 20 (4):331-341. doi:10.1051/alr:2008007

Ward AJW, Sumpter DJT, Couzin LD, Hart PJB, Krause J (2008) Quorum decision-making facilitates information transfer in fish shoals. Proceedings of the National Academy of Sciences of the United States of America 105 (19):6948-6953. doi:10.1073/pnas.0710344105

Figure 1. Frequency of the distribution of populations between two close and supposed identical FOBs

$\left(\mathrm{X}_{1} /\left(\mathrm{X}_{1}+\mathrm{X}_{2}\right)\right)$ observed in a binary choice experiment (Robert et al. 2013b).

Figure 2. Rate of leaving FOBi per unit of time, Qi, a function of the number of congener (items) under FOBi,

Xi, for the four models studied: a) Non-social model, b) Retention model, c) Departure model, d) Linear

combination of retention and departure processes. $\Theta$ (and $\Theta^{\prime}$ ) defines the maximal probability of leaving a FOB per unit of time, and K (and K') acts as a threshold. Parameter sets used: a) $\theta=0.1$ (black solid line) and $\theta=1$ (grey dashed line); b) $\theta=2, \mathrm{~K}=1$ and $\mathrm{n}=1$ (black solid line), $\mathrm{n}=2$ (grey dashed line); c) $\theta^{\prime}=1, \mathrm{~K}^{\prime}=30, \mathrm{n}^{\prime}=1$ (black solid line), n'=10 (grey dashed line); d) $\theta=2, K=0.5, \mathrm{n}=1.5, \theta^{\prime}=1, \mathrm{n}^{\prime}=100$ and $\mathrm{K}^{\prime}=20$ (black solid line) or $\mathrm{K}^{\prime}=40$ (grey dashed line).

Figure 3. Simulation outputs for $\mathrm{N}=50$. Frequency of the distribution of populations between the two FOBs $\left(\mathrm{X}_{1} /\left(\mathrm{X}_{1}+\mathrm{X}_{2}\right)\right)$, calculated using the last time step of the 500 simulations (Left panels a,c,e,g). Evolution of the number of items under both FOBs (green and red lines respectively) and in the reservoir (blue line) through time for one simulation (Right panels b,d,f,h). Parameter sets: a,b) Non-social model: $\mu=0.5, \Theta=0.5$; c,d) Retention model: $\mu=0.1, \Theta=2, K=1, n=2$; e,f) Departure model: $\mu=0.1, \Theta^{\prime}=1, K^{\prime}=20$, n’=100; g,h) Retention and departure model $\mu=0.5, \Theta=2, \Theta^{\prime}=1, K=0.5, K^{\prime}=40, n=1.5, n^{\prime}=100$. 
Figure 4. Bifurcation diagram of $X_{1} / N\left(\operatorname{or}_{2} / N\right)$ as a function of $N$. Parameter sets used: $\theta=2, K=1, \mu=0.1, n=1,2$ (grey and black respectively). Solid line indicates stable steady states and dashed line unstable steady states.

${ }^{\circ}$ Identify which mechanisms are most likely to reproduce fish aggregations dynamics under FOBs

○ Dynamics and distributions of populations of behavioral models were compared to observations

$\circ$ Results challenge the common vision theses aggregations

- Social interactions should be incorporated to reproduce the temporal patterns observed

- The characterization of these mechanisms is a priority for science-based fishery management. 


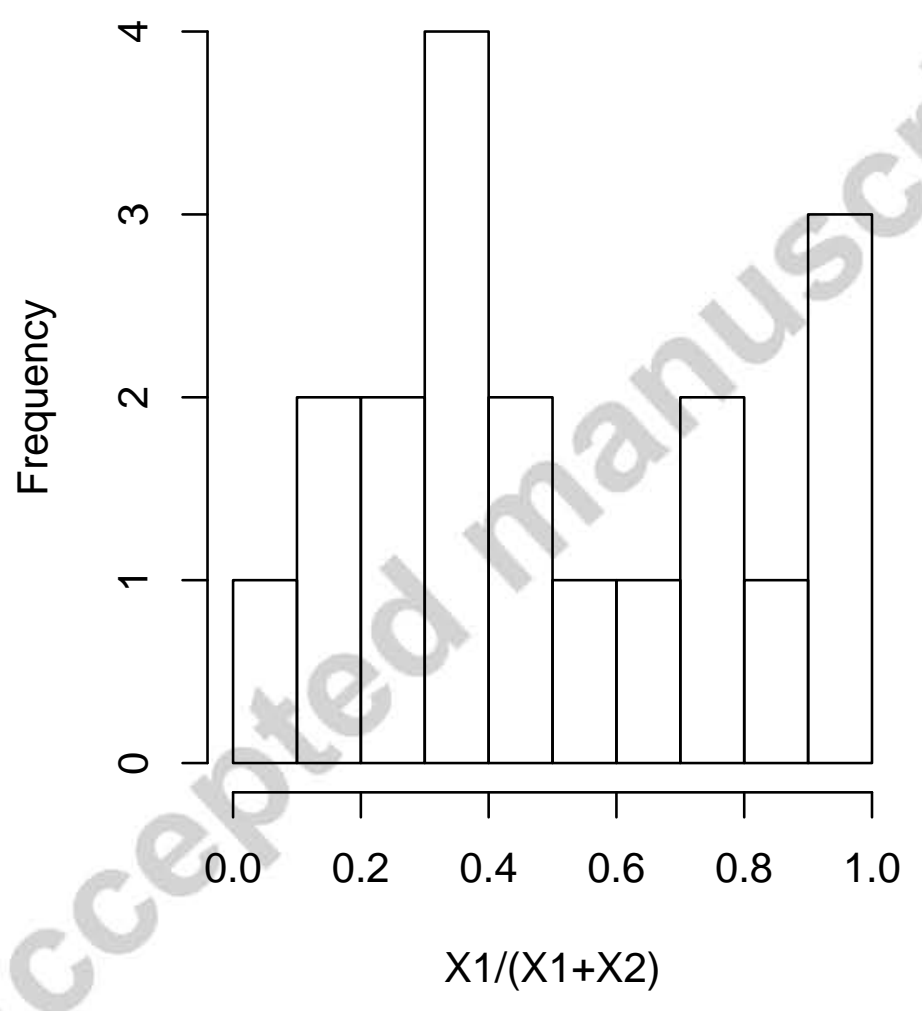



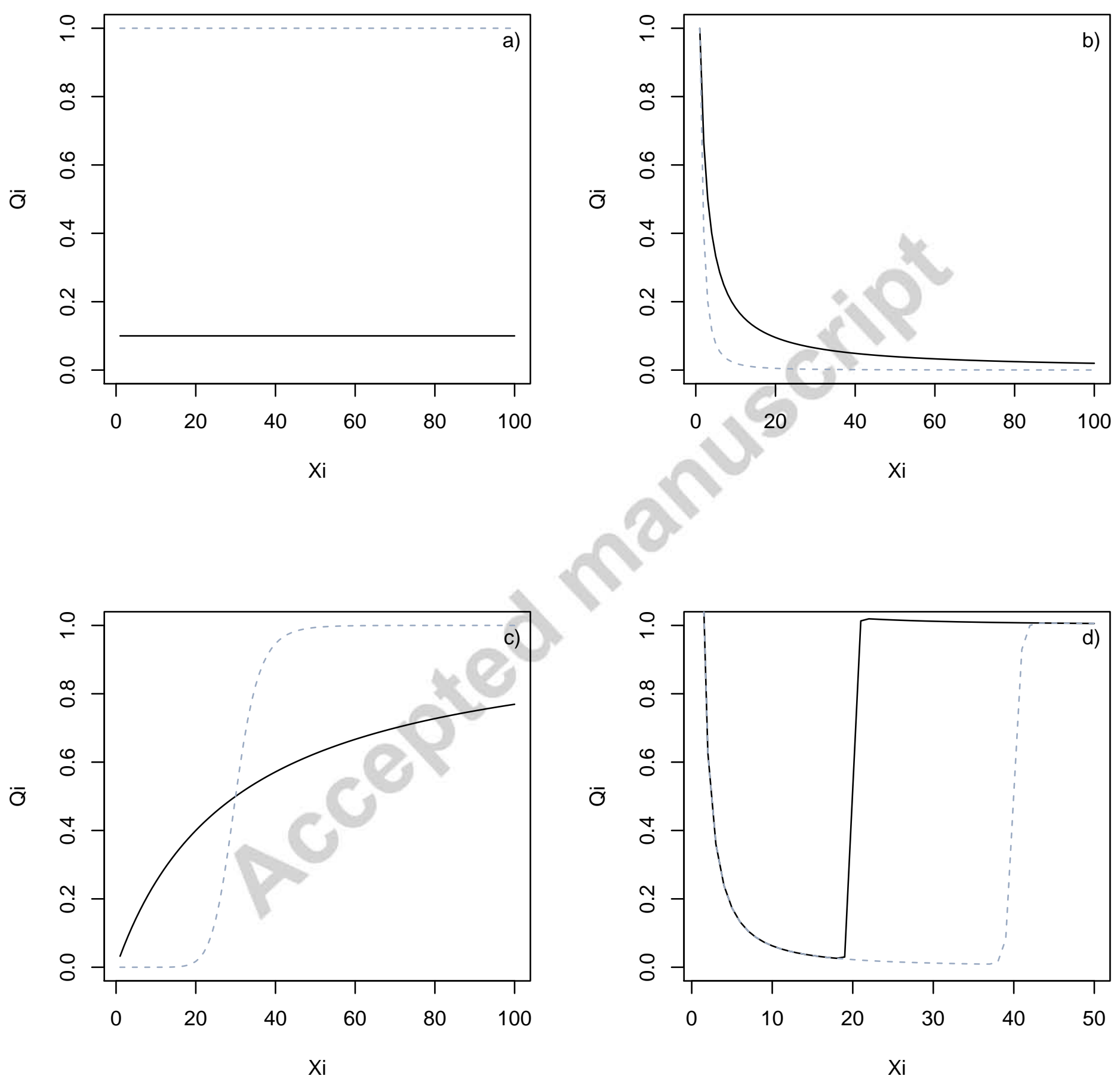

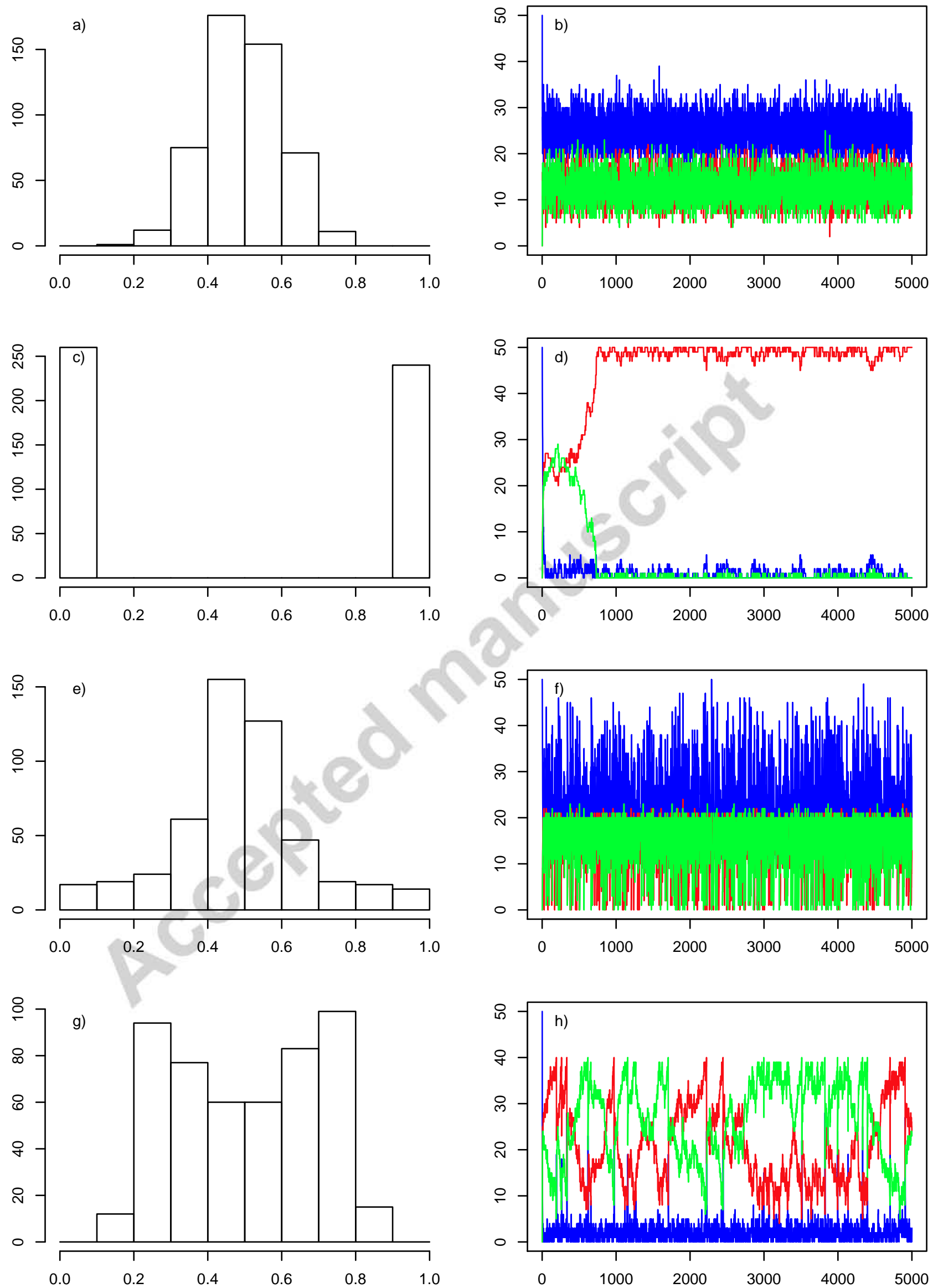


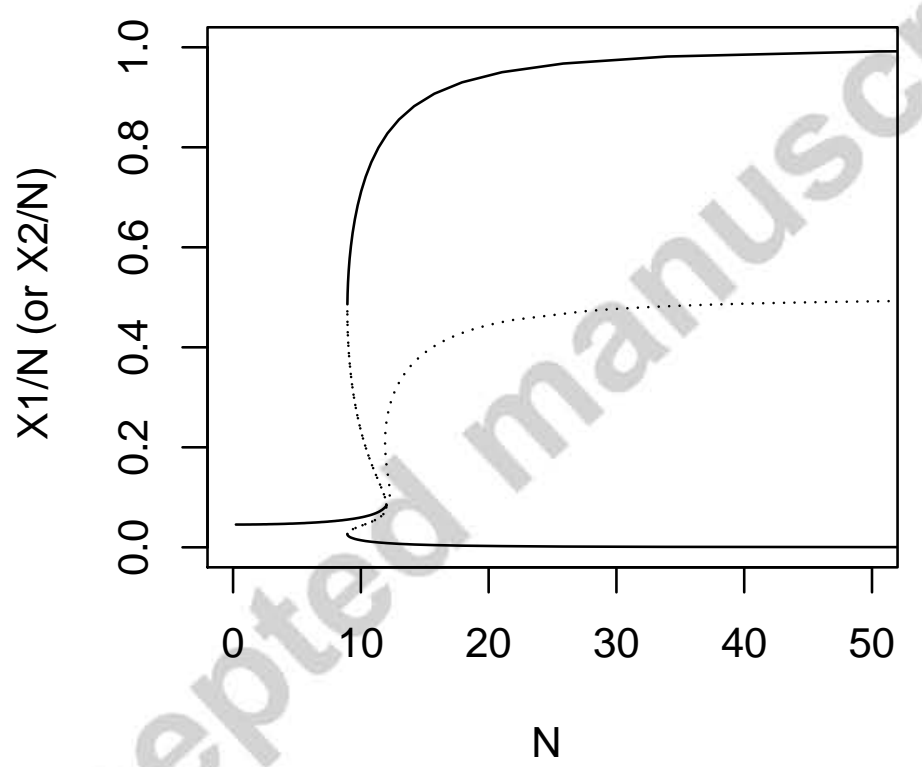

